\title{
Characterization of the human RFX transcription factor family by regulatory and target gene analysis
}

Debora Sugiaman-Trapman ${ }^{1}$, Morana Vitezic ${ }^{2}$, Eeva-Mari Jouhilahti ${ }^{1}$, Anthony Mathelier ${ }^{3,4,5}$, Gilbert Lauter ${ }^{1}$, Sougat Misra ${ }^{6}$, Carsten O. Daub ${ }^{1,7}$, Juha Kere ${ }^{1,8,9+}$ and Peter Swoboda ${ }^{1 *+}$ (D)

\begin{abstract}
Background: Evolutionarily conserved RFX transcription factors (TFs) regulate their target genes through a DNA sequence motif called the X-box. Thereby they regulate cellular specialization and terminal differentiation. Here, we provide a comprehensive analysis of all the eight human $R F X$ genes (RFX1-8), their spatial and temporal expression profiles, potential upstream regulators and target genes.

Results: We extracted all known human RFX1-8 gene expression profiles from the FANTOM5 database derived from transcription start site (TSS) activity as captured by Cap Analysis of Gene Expression (CAGE) technology. RFX genes are broadly (RFX1-3, RFX5, RFX7) and specifically (RFX4, RFX6) expressed in different cell types, with high expression in four organ systems: immune system, gastrointestinal tract, reproductive system and nervous system. Tissue type specific expression profiles link defined RFX family members with the target gene batteries they regulate. We experimentally confirmed novel TSS locations and characterized the previously undescribed RFX8 to be lowly expressed. RFX tissue and cell type specificity arises mainly from differences in TSS architecture. RFX transcript isoforms lacking a DNA binding domain (DBD) open up new possibilities for combinatorial target gene regulation. Our results favor a new grouping of the RFX family based on protein domain composition. We uncovered and experimentally confirmed the TFS SP2 and ESR1 as upstream regulators of specific RFX genes. Using TF binding profiles from the JASPAR database, we determined relevant patterns of X-box motif positioning with respect to gene TSS locations of human RFX target genes.
\end{abstract}

Conclusions: The wealth of data we provide will serve as the basis for precisely determining the roles RFX TFs play in human development and disease.

Keywords: Cell differentiation, Cilia, Spermatogenesis, Immune cell proliferation, Neuronal development, Cell cycle control, Tumor suppression

\section{Background}

RFX (Regulatory Factor binding to the X-box) transcription factors (TFs) share and are defined by a conserved, specialized winged-helix type DNA binding domain (DBD) [1]. RFX genes have been identified in all animals within the Unikont branch of eukaryotes, which excludes algae, plants and various protozoan branches [2]. Metazoan genomes encode one to several RFX genes. C. elegans possesses one,

\footnotetext{
* Correspondence: peter.swoboda@ki.se

${ }^{\dagger}$ Equal contributors

${ }^{1}$ Department of Biosciences and Nutrition, Karolinska Institutet, Huddinge, Sweden

Full list of author information is available at the end of the article
}

Drosophila has two $[3,4]$, mammals have eight and - due to genome duplication - fishes have nine $R F X$ genes [2, 5-10]. Human RFX1-7 have previously been described [9], while RFX8 (ENSG00000196460, www.ensembl.org) has not been characterized.

In different organisms, RFX TFs have been shown to regulate genes involved in various and seemingly disparate cellular and developmental processes [7] like the cell cycle and DNA repair [11, 12], or aspects of cellular differentiation, like the functional maturation of cells of the immune response [13] and the development of cilia on the surface of polarized cells [14-16]. As a consequence of these roles 
in development, mutations in RFX genes can lead to severe disease states. Mutations in RFX5 cause autosomal recessive Bare Lymphocyte Syndrome (OMIM \#209920), characterized by severe combined immunodeficiency due to failure in HLA expression. Mutations in RFX6 cause autosomal recessive Mitchell-Riley Syndrome, characterized by neonatal diabetes and malformations of the gut (OMIM \#615710). Rfx mutant mice exhibit a plethora of mild to fatal phenotypes, ranging from male sterility [17] to brain abnormalities [18]. These phenotypes are often attributed to cilia dysfunction [17, 19-21]. Of note, many human ciliopathy genes are strongly assumed to be RFX TF targets, given that their orthologs have been shown to be RFX TF targets in several different organisms, ranging from C. elegans to mouse [22-24].

In addition to the DBD, RFX TFs may contain other conserved domains like activation (AD) and dimerization (DIM) domains and the domains $\mathrm{B}$ and $\mathrm{C}$ of unknown function [7, 9]. The RFX DBD recognizes an imperfect inverted repeat sequence, the X-box motif, to which it binds [1]. RFX TF binding to the X-box motif has repeatedly been demonstrated by using methods ranging from in vitro binding studies, in vivo expression and mutation analyses to SELEX and ChIP sequencing approaches [8, 25-28]. Combined, these approaches led to the discovery of large batteries of RFX target genes [16].

By contrast, very little is known about upstream regulators of RFX genes. So far only a few studies in mice, zebrafish and flies have identified TFs of the bHLH class, Neurog3 and Atonal, as well as the homeobox protein Noto as upstream regulators of RFX genes [29-31]. In the yeast $S$. cerevisiae an upstream phosphorylation cascade controls expression of the RFX gene Crt1 [32].

In this study - using extensive analysis of data from the FANTOM5 database followed by experimental validations - we present an in-depth characterization of the entire human $R F X$ gene family (RFX1-8), including the previously undescribed $R F X 8$ and $R F X$ transcript isoforms that encode TFs without DBD. We provide an updated grouping of human RFX TFs and show that RFX functional domain composition is independent of expression profile. Our exhaustive analysis of $R F X$ expression in many different human tissues and cell types suggests that RFX tissue and cell type specificity arises mainly from differences in TSS architecture and not from different transcript isoforms. We determined with high precision the positioning of X-box motifs with respect to TSS locations of human RFX target genes. Using cluster analysis based on tissue and cell type specific expression profiles we link defined RFX family members with the target gene batteries they regulate. Further, we provide a first list of candidate upstream regulators of human $R F X$ genes. The wealth of data we provide will serve as the basis for future studies of the role of RFX TFs in human development and disease.

\section{Results}

Expression of human RFX genes in different tissue types Detailed expression profiles of the human $R F X 1-8$ genes have not been described. We used data from the FANTOM5 database that is based on experimental expression profiling by CAGE technology across a wide spectrum of human biological samples. The expression level of a given CAGE TSS location is defined by an arbitrary unit, tags per million (TPM) [33]. We extracted 37 CAGE TSS locations for RFX1-8 from the FANTOM 5database and shortlisted these to 30 TSS locations by merging those which are in close proximity to each other and have similar expression profiles (cf. Methods). We then named these 30 TSS locations alphabetically, whereby promoter A (pA) is the highest expressed TSS. Expression of each RFX TSS is described in detail for human tissues, primary cells and cell lines (Additional file 1). The wealth of biological samples allows classifying the expression profiles for human RFX1-8 in different cell types.

A given RFX TSS location is considered as being expressed broadly if it is expressed at TPM $>5$ in a large number and variety of tissues $(n>10)$. Conversely, a given RFX TSS location is considered as being expressed specifically if it is expressed at TPM $>5$ in a small number of tissues of the same organ $(n<10)$. We found most TSS locations of RFX1-3, 5 and 7 to be expressed broadly in many tissue types whereas the TSS locations of RFX4 and RFX6 are all expressed in specific tissue types.pA@RFX4 is highly specific in brain and spinal cord tissues, while pB and pC@RFX4 are highly specific in testis. RFX6 TSS locations are all specifically expressed in the gastrointestinal tract (GI) (Additional file 2). We performed hierarchical clustering of the 30 RFX TSS locations based on their expression values (TPM) across 135 human tissue samples. Thereby we identified four major tissue clusters, namely immune system [34], gastrointestinal tract [35, 36], testis [37] and brain and spinal cord [18, 38-41], and two minor clusters, namely uterus and lung [42] (Additional file 2).

The expression of RFX8 is very low, making it the most elusive member of the human RFX family that has hitherto avoided detection. Here we identified RFX8 TSS locations with highest expressions in some tissues of the immune system $(\mathrm{pA}, \mathrm{pC}, \mathrm{pD})$ and the gastrointestinal tract $(\mathrm{pB}, \mathrm{pE})$. However, the tissue expression values for $\mathrm{pC}, \mathrm{pD}$ and $\mathrm{pE}$ were hard to distinguish from background noise $(\mathrm{TPM}<1)$. In primary cells and cell lines, RFX8 TSS locations had higher expression values, with the most prominent expression in a Schwannoma cell line (Additional file 2).

\section{Connecting TSS expression profiles to protein-coding transcript isoforms}

FANTOM5 data allowed us to determine TSS locations and expression profiles. In order to connect the 30 RFX TSS 
locations described above to known transcript isoforms, we set a maximum distance limit of 50 nt between the TSS location and the nearest Ensembl protein-coding transcript with a complete open reading frame. We found that 18 of these 30 RFX TSS locations matched Ensembl proteincoding transcripts. The remainder (12) of the 30 RFX TSS locations were treated as novel transcript isoforms in human tissues. We selected seven of these as representatives for experimental validation by RT-PCR and sequencing (Table 1 , Additional file 3: Table S1). The seven novel transcripts consist of (i) testis specific pC@RFX1 and pE@RFX3, (ii) broadly expressed and highest in brain pC@RFX3,
pC@RFX5, pA@RFX7, pC@RFX7, and (iii) lowly expressed pA@RFX8.

Next, we assessed the full transcript sequences (from $5^{\prime}$ to $3^{\prime}$ UTRs) including their coding potential (from start to stop codons) from both the matched Ensembl protein-coding transcripts and the novel sequenceverified transcripts (Table S2 in Additional file 2). Representatives of the RFX1-8 transcripts are shown in Fig. 1a. We found that the majority of RFX transcript isoforms originating from the same gene encode identical proteins suggesting that tissue and cell type specificity arises mainly from differences in TSS architecture and

Table 1 RFX1-8 expression data and novel transcripts

\begin{tabular}{|c|c|c|c|c|}
\hline \multirow[t]{2}{*}{ Gene (chromosome) } & \multirow[t]{2}{*}{ TSS } & \multirow{2}{*}{$\begin{array}{l}\text { Matched } \\
\text { Ensemble } \\
\text { transcript }\end{array}$} & \multicolumn{2}{|l|}{ Tissue profile summary } \\
\hline & & & Expression & Highest in \\
\hline \multirow[t]{3}{*}{ RFX1 (chr19) } & pA@RFX1 & ENST00000254325 & Broad & Cerebellum (brain) \\
\hline & pB@RFX1 & ENST00000254325 & & \\
\hline & pC@RFX1* & Novel transcript* & Specific & Testis \\
\hline \multirow[t]{3}{*}{ RFX2 (chr19) } & pA@RFX2 & ENST00000303657 & Broad & Uterus \\
\hline & pB@RFX2 & ENST00000303657 & & Testis \\
\hline & pC@RFX2 & ENST00000303657 & & Medulla oblongata (brain) \\
\hline \multirow[t]{5}{*}{ RFX3 (chr9) } & pA@RFX3 & ENST00000382004 & Broad & Cerebellum (brain) \\
\hline & pB@RFX3 & ENST00000382004 & & Lung, fetal \\
\hline & pC@RFX3* & Novel transcript* & & Cerebellum (brain) \\
\hline & pD@RFX3 & ENST00000382004 & & Lung, fetal \\
\hline & $\mathrm{pE} @ R F X 3^{*}$ & Novel transcript* & Specific & Testis \\
\hline \multirow[t]{3}{*}{ RFX4 (chr12) } & pA@RFX4 & ENST00000392842 & Specific & Spinal cord \\
\hline & pB@RFX4 & ENST00000229387 & Specific & Testis \\
\hline & pC@RFX4 & ENST00000357881 & & \\
\hline \multirow[t]{4}{*}{ RFX5 (chr1) } & pA@RFX5 & ENST00000290524 & Broad & Blood (immune system) \\
\hline & pB@RFX5 & ENST00000290524 & & Tonsil (immune system) \\
\hline & pC@RFX5* & Novel transcript* & & Brain, fetal \\
\hline & pD@RFX5 & Novel transcript & & Duodenum, fetal (Gl) \\
\hline \multirow[t]{3}{*}{ RFX6 (chr6) } & pA@RFX6 & ENST00000332958 & Specific & Duodenum, fetal (Gl) \\
\hline & pB@RFX6 & ENST00000332958 & & \\
\hline & pC@RFX6 & ENST00000332958 & & \\
\hline \multirow[t]{4}{*}{ RFX7 (chr15) } & $\mathrm{pA} @ R F X 7^{*}$ & Novel transcript* & Broad & Cerebellum (brain) \\
\hline & pB@RFX7 & ENST00000559447 & & \\
\hline & $\mathrm{pC} @ \mathrm{RFX7}^{*}$ & Novel transcript* & & \\
\hline & pD@RFX7 & Novel transcript & & \\
\hline \multirow[t]{5}{*}{ RFX8 (chr2) } & pA@RFX8* & Novel transcript* & Lowly expressed $($ TPM $<5)$ & Thymus (immune system) \\
\hline & pB@RFX8 & ENST00000428343 & & Medial frontal gyrus (brain) \\
\hline & pC@RFX8 & Novel transcript & Noise $($ TPM < 1) & Heart \\
\hline & pD@RFX8 & Novel transcript & & Breast \\
\hline & $\mathrm{pE} @ R F X 8$ & Novel transcript & & Rectum, fetal \\
\hline
\end{tabular}

Thirty TSS locations from eight human RFX genes and their respective tissue profile summaries are presented (cf. Methods; Gl = gastrointestinal tract). For an expanded summary and the analysis of functional domains, see Tables S1 and S2 in Additional file 3, respectively. Novel transcripts are marked in bold and those selected for experimental validation are marked with an asterisk. For RT-PCR verified sequences of novel transcripts, see Table S9 in Additional file 3 


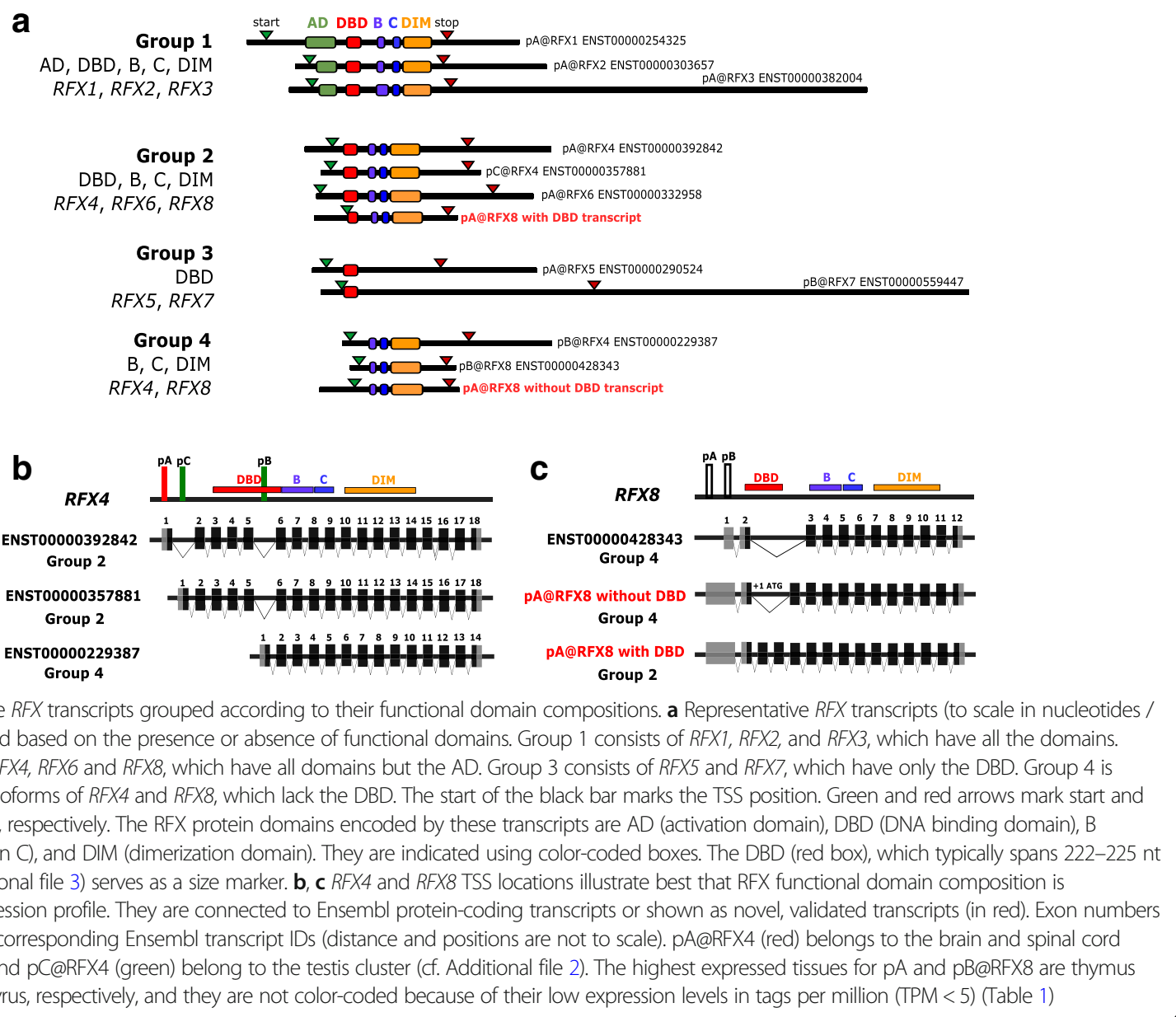

regulation through their corresponding promoters. The exceptions are RFX1, RFX4 and RFX8 with isoforms encoding different protein variants. The testis specific pC@RFX1 transcript isoform encodes a shortened Nterminal region upstream of the activating domain (AD). RFX4 transcript isoforms have been extensively studied [43-45] and thus complement our results, where we found isoforms encoding different RFX4 protein variants. The RFX8 gene encodes a TF protein lacking a DNA binding domain (DBD) (ENSP00000401536, www.ensembl.org). Here, we experimentally validated RFX8 transcripts by sequencing cDNA from human brain total RNA and uncovered novel splicing patterns leading to alternative RFX8 protein variants, with and without DBD (Table S1 in Additional file 3).

\section{RFX functional domain composition is independent of expression profile}

Human RFX TFs were previously categorized through phylogenetic analysis of their four functional domains outside the DBD: activating domain (AD), domain $\mathrm{B}$, domain $\mathrm{C}$ and dimerization domain (DIM) $[9,10,16]$.
Given the variation in coding potential of all 30 RFX1-8 transcript isoforms, we investigated whether there is a correlation between the presence or absence of certain RFX functional domains and the CAGE TSS expression profiles in human tissues. First, we categorized all RFX1-8 transcripts into four groups based on their functional domain structure: (i) Group 1: RFX1-3 with all known domains, (ii) Group 2: RFX4, RFX6 and RFX8 lacking the AD, (iii) Group 3: RFX5 and RFX7 with only the DBD, (iv) Group 4: RFX4 and RFX8 lacking the DBD (Fig. 1a). When we then compared these four groups to their respective TSS expression profiles, we did not find any indication that RFX TFs with similar domain composition would be expressed broadly or specifically in a certain tissue cluster, suggesting that the RFX functional domain composition is independent of expression profile. We analyzed RFX4 and RFX8 in more detail to illustrate this point (Fig. 1b, c).

Based on the FANTOM5 expression profiles, the gene RFX4 is highly tissue specific compared to other RFX genes. In our analysis, we connected three RFX4 TSS 
locations to three different Ensembl protein-coding transcripts (Fig. 1b). The longest, highest expressed transcript falls into Group 2 (lacking the AD); it is specifically expressed in the brain and spinal cord. The other two less expressed transcripts are both testis specific, whereby one belongs to Group 2 (lacking the AD) and the other one to Group 4 (lacking the DBD). The newly described gene RFX8 is the least expressed of all the RFX genes. We connected the two highest expressed RFX8 TSS locations with three possible transcripts (Fig. 1c). The same TSS can lead to different transcripts encoding protein variants, which either fall into Group 2 (lacking the $\mathrm{AD}$ ) or Group 4 (lacking the $\mathrm{DBD}$ ), suggesting an additional layer of gene regulation on top of the TSS architecture itself. Interestingly, RFX8 transcripts with DBD revealed that the RFX8 DBD is slightly shorter than the DBDs in RFX1-7. Whereby, multiple sequence alignments of RFX DBD amino acid sequences reveal that it is the least conserved $\mathrm{N}$-terminal amino acids of the DBD that are missing in RFX8 (Figure S1 in Additional file 3).
Tissue and cell type specific clustering of RFX family members with the target genes they regulate

To correlate and eventually predict which RFX family member regulates which target gene in which human tissue and cell type, we compared and clustered the expression profiles of all RFX family members with direct RFX target genes. We selected from the literature a large number of validated direct RFX target genes in humans, as demonstrated either by a biochemical interaction between an RFX TF and the respective X-box promoter motif or by confirmation of the X-box function by mutation analysis (Table S3 in Additional file 3). We then extracted the CAGE TSS expression values (TPM) of these genes from the FANTOM5 database and performed unsupervised heat map clustering based on the correlations of expression values across 135 tissue types (Fig. 2).

We identified strong tissue specific $R F X$ and target gene clusters, namely for testis (RFX1-4 and target genes GPR56, ALMS1, RFX1) and the gastrointestinal tract (RFX5-6 and target gene INS/insulin). We also observed strong differences between clusters of the

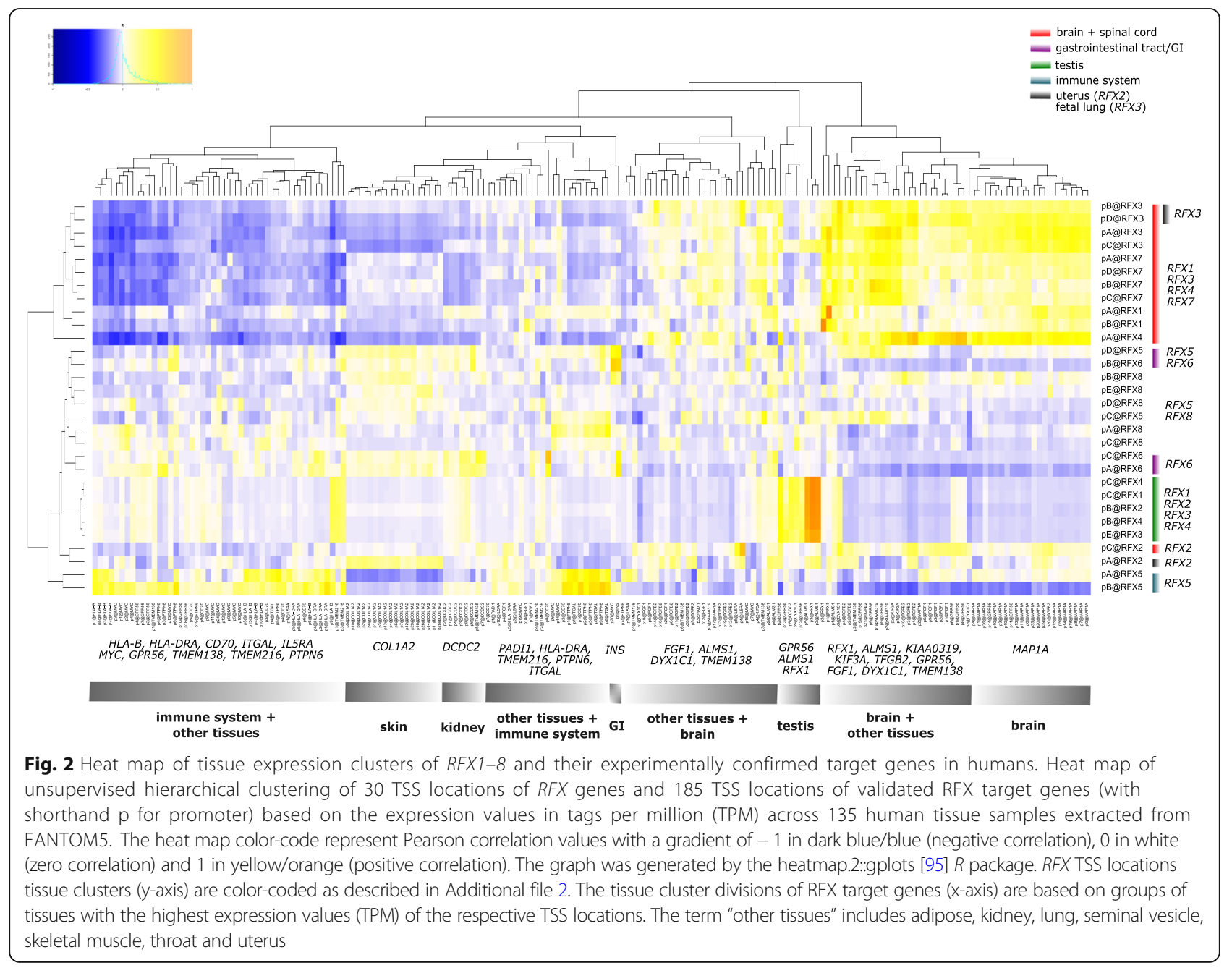


immune system (RFX5) and the brain (RFX1, 3, 4, 7). This underscores that (i) the respective RFX family members regulate different sets of target genes as they are not co-expressed in a given tissue type, and (ii) for a given target gene RFX TFs can act as activators or as inhibitors. For brain tissue and cell types, RFX1, 3, 4 and 7 clustered tightly together, indicating a preference for these RFX family members to (co-) regulate the expression of brain-specific genes such as the ciliopathy/ Alström syndrome gene $A L M S 1$, the dyslexia candidate gene KIAA0319 or the gene MAP1A. Interestingly, another member of the brain cluster, pC@RFX2 (Table 1, Additional file 2), in the context of target genes clustered separately (Fig. 2), suggesting that in the brain RFX2 regulates a distinct set of target genes. Alternatively, RFX2 may interact with other RFX family members or other co-factors without preference as long as they are co-expressed in a given tissue and cell type.

\section{X-box motif positioning in the human genome}

RFX TFs regulate their target genes by binding to a conserved X-box motif in the promoter region. Previous X-box motif searches have typically been carried out using 1-3 kb sequence windows upstream of the TSS or ATG, such as in C. elegans [27], D. melanogaster [23], mouse and human [46]. To our knowledge, precise X-box motif positioning has not been characterized in the human genome. Thus, we determined the most likely positioning of functional X-box motifs in the promoter region, defined as 5000 bp upstream $(-5000)$ and 2000 bp downstream $(+2000)$ in relation to TSS locations, of experimentally validated human RFX target genes.

To facilitate the search, we used two curated TF binding profiles for human RFX available in the JASPAR (2018) database [47]: RFX2 (MA0600.1) and RFX5 (MA0510.1) (Table S4 in Additional file 3). As a control, we selected a 10-fold larger random set of TSS locations across the human genome. Our search effort revealed that X-box hits are typically located very close to RFX target genes TSS locations (Fig. 3, Table S5 in Additional file 3). Based on search and find statistics the $\mathrm{X}$-box positioning window can be further subdivided into a robust window of -500 to $+500 \mathrm{bp}$ and a permissive window of -2300 to + $1400 \mathrm{bp}$. Using an independent search approach (the MEME suite FIMO software) [48] we confirmed these overall search and find parameters for human X-box motifs. Our analysis enhances the prediction power of future searches for functional X-box motifs, which relates to both upstream and downstream of TSS locations of candidate human RFX target genes and pinpoints their likely locations. Functional X-box motifs at larger distances from TSS locations (e.g at distal enhancers) are likely to be the exception rather than the norm (Table S6 in Additional file 3).

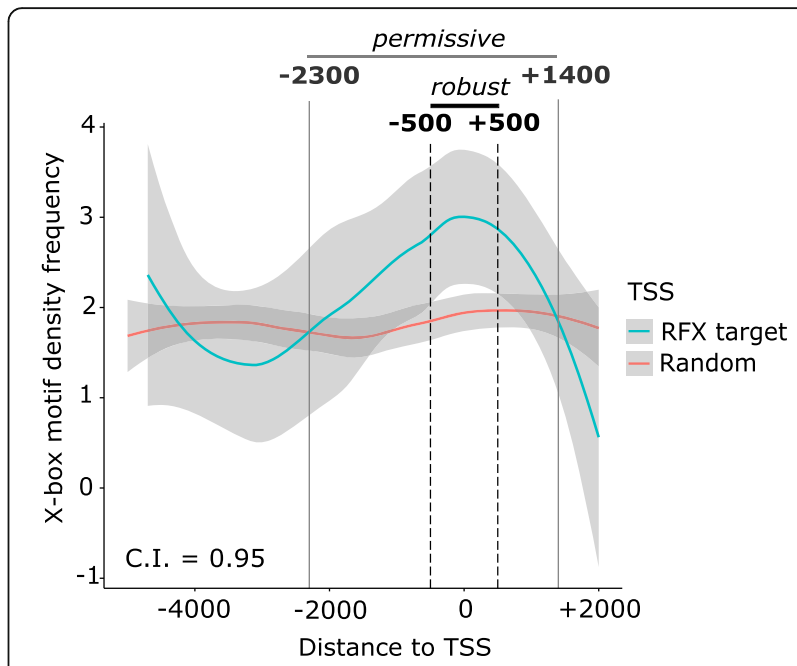

Fig. 3 X-box motif position with respect to TSS locations. Density frequency of X-box motif positions with respect to the TSS locations of experimentally proven direct RFX target genes in humans (shown in blue) and a set of $10 \times$ random TSS locations from FANTOM5 (shown in red): TSS -5000 to +2000 bp windows were scanned with two JASPAR RFX motifs (RFX2 MA0600.1 and RFX5 MA0510.1) with 80\% threshold. We define a sequence window as "robust" by the area where the two curves with 95\% C.I. smoothing do not overlap. We define a sequence window as "permissive" by the area where the two curves intersect

Prediction of upstream RFX regulators using transcription factor binding site (TFBS) analysis

Identifying the upstream regulators of RFX genes will allow predicting the developmental and cellular niche that RFX TFs occupy. Thus, we searched for TF binding profiles over-represented in the promoter and enhancer regions of all 8 human $R F X$ genes. We used search windows of 5000 to +2000 bp in relation to 30 RFX TSS locations and - 200 to +200 bp around the midpoints of 13 significantly correlated candidate RFX enhancer sequences (extracted from Andersson et al. [49]; Table S7 in Additional file 3). We then scanned these regions with all the core vertebrate TF binding profiles present in the JASPAR 2016 database [47]. The enrichment for TF binding profiles was assessed against a 10-fold larger random set of human promoter and enhancer regions using the oPOSSUM3 tool [50].

We identified 19 over-represented TF binding profiles (Fig. 4) associated to the TFs SP2 (specificity protein 2) (JASPAR profile MA0516.1), E2F4 (E2 factor 4) (MA0470.1), KLF16 (Kruppel like factor 16) (MA0741.1), SP8 (specificity protein 8) (MA0747.1), SP3 (specificity protein 3) (MA0746.1), EGR3 (early growth response 3) (MA0732.1), ESR1 (estrogen receptor alpha) (MA0112.3), Creb5 (cAMP responsive element binding protein 5) (MA0840.1), ZNF740 (zinc finger protein 740) (MA0753.1), ATF7 (activating transcription factor 7) (MA0834.1), SOX21 (sex determining region Y-box 21) (MA0866.1), MZF1 (myeloid zinc finger 1) (MA0056.1 and MA0057.1), Tcfl5 (transcription factor like 


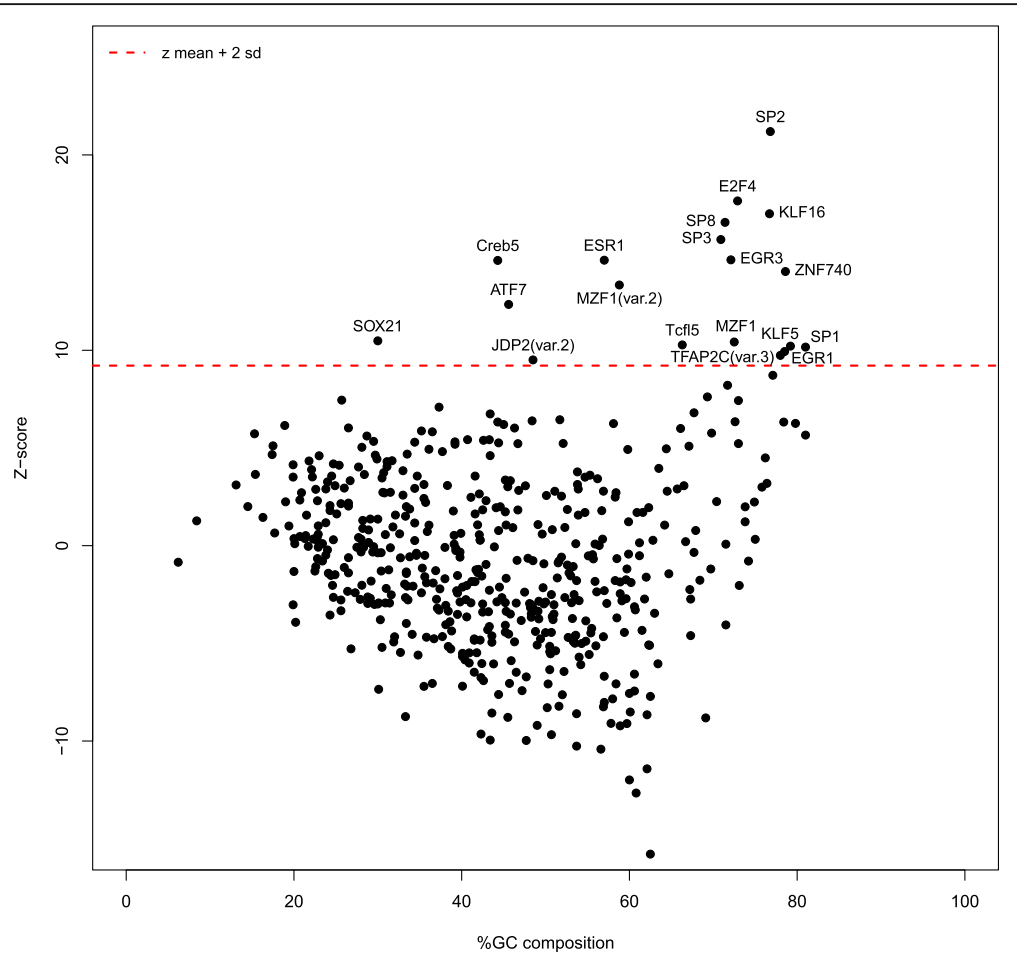

Fig. 4 TF binding profiles in the promoter and enhancer regions of RFX genes. Distribution of all the $z$-scores of all the core vertebrate transcription factor binding site (TFBS) profiles in JASPAR 2016, with the search areas consisting of -5000 to +2000 bp with respect to the 30 RFX TSS locations and $-200 \mathrm{bp}$ to $+200 \mathrm{bp}$ from the mid-points of the RFX enhancers, against a background of a set of 10X random TSS locations and enhancers with identical window size and matching \%GC distribution from FANTOM5. High-scoring or over-represented TF binding site profiles were computed as having $z$-scores above the mean $+2 \times$ standard deviation (red dotted line)

5) (MA0632.1), KLF5 (Kruppel like factor 5) (MA0599.1), SP1 (specificity protein 1) (MA0079.3), EGR1 (early growth response 1) (MA0162.2), TFAP2C (transcription factor AP-2 gamma) (MA0815.1) and JDP2 (Jun dimerization protein 2) (MA0656.1). The full list of the TF binding profiles can be found in Additional file 4.

\section{siRNA validation of RFX regulators}

To test if any of the over-represented TF binding profiles can be linked to functional upstream regulation of RFX genes, we selected two TFs within the high-scoring TF binding profiles. We used siRNA knockdown of SP2 and ESR 1 followed by qRT-PCR measuring the fold change of mRNA expression levels of RFX genes. We could successfully demonstrate knockdown of SP2 and ESR1 both at the mRNA level by qRT-PCR and at the protein level by immunoblotting (Fig. 5). The TF binding profiles of SP2 and ESR1 both scored clearly above the z-score threshold (Fig. 4). We selected the human MCF7 breast cancer cell line for which data are available in FANTOM5. In this cell line the genes SP2, ESR1, RFX1-3, -5 , and -7 are expressed sufficiently high $(\mathrm{TPM}>5)$, while the genes RFX4, -6 and -8 are not expressed (TPM $=0)$. We used efficiency-adjusted fold change quantification against scrambled (Scr) control siRNA normalized to the geometric mean of HPRT1 and HSPCB as two independent reference genes [51]. All the Ct levels of the test siRNA and Scr control siRNA can be found in Additional file 5.

We observed that siRNA knockdown of SP2 and ESR1 in human MCF7 cells resulted in a significant fold change in the mRNA expression levels of at least one of the RFX genes (Fig. 5). siRNA knockdown of SP2 resulted in both activating and inhibiting effects on $R F X$ genes, whereby only $R F X 7$ showed significant up-regulation. In contrast, siRNA knockdown of ESR1 revealed consistent inhibitory effects on all the RFX genes analyzed, with RFX2, - 3, -5, and -7 being significantly up-regulated. These data show that computational TFBS analyses of the promoter regions of RFX1-8 correctly identified functional upstream regulators of these $R F X$ genes. Depending on the individual $R F X$ gene these upstream regulators act either as activators or as repressors.

\section{Discussion}

We have exhaustively analyzed all eight members of the human RFX TF gene family (RFX1-8). By extracting and computationally analyzing large-scale experimental data sets, we were able to describe in detail RFX gene expression as well as the $R F X$ gene regulatory landscape in many different human tissues and cell types, including 


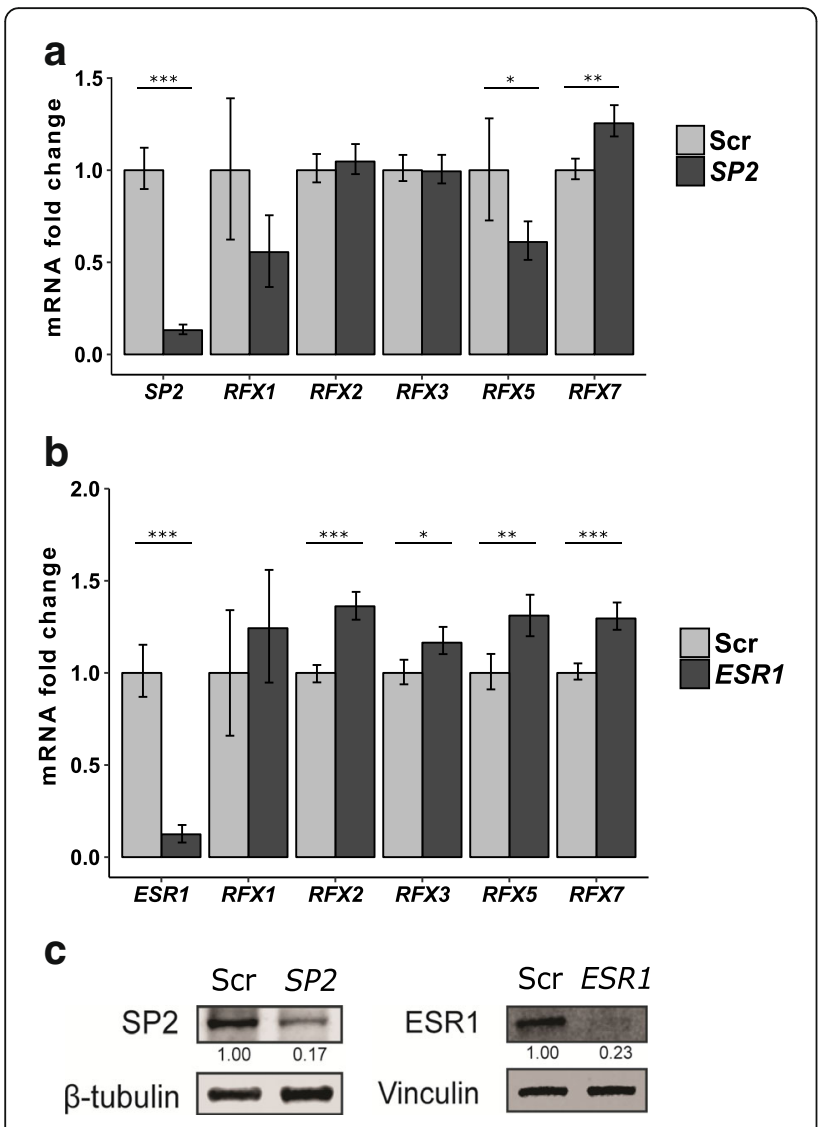

Fig. 5 siRNA validation of candidate RFX regulators. The genes $S P 2$ and ESR1 represent the high-scoring group of candidate $R F X$ regulators (cf. Fig. 4 and Additional file 4). In the MCF7 breast cancer cell line, amplification efficiency-adjusted mRNA fold change quantifications of $R F X 1,2,3,5$ and 7 were normalized to the geometric mean of HPRT1 and $H S P C B$, whereby a fold change equaling 1 describes an unchanged expression level. For this we used (a) SP2 siRNA versus scrambled (Scr) control siRNA knockdown, and (b) ESR1 siRNA versus scrambled (Scr) control siRNA knockdown. In (a, b) error bars represent SEM and foldchange statistical significance was calculated using the student twosample t-test ( ${ }^{* * *} p$-value $\leq 0.01,{ }^{* *} p$-value $\leq 0.05,{ }^{*} p$-value $\left.\leq 0.1\right)$. a, c SP2 siRNA knockdown was confirmed at both the mRNA and protein level and a significant up-regulation of RFX7 and down-regulation of RFX5 were observed. b, c ESR1 siRNA knockdown was confirmed at both the mRNA and protein level and significant up-regulations of $R F X 2,3,5$ and 7 were observed. c Immunoblotting band intensities were quantified using ImageJ and normalized with the indicated loading controls

in parts the experimental validation thereof. We provide the first detailed experimental characterization of RFX8 and of $R F X$ isoforms without DBD. Further, we provide insight into upstream regulators of human RFX genes and determined the sequence windows in which - in most cases - human RFX TFs act as direct regulators of their target genes. Thereby we provide an in-depth catalogue and key resource for future work on the roles that RFX TFs play in human development and disease.

Our extensive survey of all the human RFX (1-8) gene expression profiles enabled us to carefully analyze all the transcript isoforms and determine the potential protein variants encoded by these isoforms. We ordered all expression profiles from low to high, from broad to tissue specific, made tissue and cell type assignments, including isoform correlations and non-correlations, and thereby were able to cluster the expression profiles for all the isoforms of all the human $R F X$ genes. We found and experimentally validated that - typically - RFX gene TSS locations (of the same gene) would lead to the same protein variant, suggesting that it is mostly promoter and TSS architecture that gives rise to diversity in gene expression profiles. These results highlight the importance of studying non-coding regulatory regions of key genes involved in developmental processes such as cell type specification and differentiation. Exceptions include TSS locations for the gene RFX4 that were spread across a large genomic distance leading to transcript isoforms encoding different tissue specific protein variants.

Our work lead to an updated grouping of human RFX TFs and showed that RFX functional domain composition is independent of expression profile. We identified two RFX genes, RFX4 and RFX8, which can encode protein variants without DBD. The function of RFX protein variants without DBD is unclear. Possibly, they act as tissue-specific co-repressors, similar to SHP proteins [52]. This potential role is clearly inferred for RFX4, where such competitive co-repression may occur in the testis but not in the nervous system [44]. Transcript validation for the newly described gene $R F X 8$ revealed the possibility for encoding protein variants with and without a DBD. For the protein variant with DBD, this domain would be slightly shorter as it is missing the least conserved $\mathrm{N}$-terminal 20 amino acids. In addition to the overall low expression level of $R F X 8$, it raises the question of RFX8 functionality. RFX8 was most prominently expressed in Schwannoma cells, suggesting a role for RFX8 in Schwann cell proliferation.

Given the central role that RFX TFs play during development (e.g. in the differentiation of cilia), we were interested in finding candidate upstream regulators of RFX genes. We used computational predictions based on over-represented TF binding profiles to find candidate upstream regulators of $R F X$ genes and thereby infer the developmental pathways that RFX1-8 are part of. The over-represented TF binding profiles that our analysis uncovered are associated with TFs involved in (i) neural development (SP2, ESR1, Creb5, SOX21) [53-56] and neurite outgrowth (KLF16, EGR3) [57, 58], (ii) cognitive functions (EGR3, EGR1) [59], (iii) craniofacial development (SP8) [60], (iv) proliferation of immune cells (EGR3, KLF5) [61, 62], platelet formation (SP3, SP1) [63] and innate immunological memory (ATF7) [64], (v) cell cycle control (E2F4) [65, 66] and tumor suppression (ZNF40, MZF1, TFAP2C, JDP2) [67-70], and (vi) reproductive functions (ESR1, Tcfl5) [54, 71]. TF binding 
profiles for RFX TFs themselves were not over-represented, suggesting that autoregulation is not a common feature for the expression of RFX1-8 genes and that RFX1 autorepression may be the only exception [72].

We validated SP2 and ESR1 by siRNA knockdown and qRT-PCR and found that they act as inhibitors of the $R F X$ genes. We assume that these candidate upstream regulators act directly, given the over-representation of their TF binding site profiles in $R F X 1-8$ promoter and candidate $R F X$ enhancer regions. The cellular context we used for experimental validation, human MCF7 breast cancer cells, very likely does not represent all human tissues. Thus, more exact mechanisms of $R F X$ gene regulation remain to be analyzed in different cell-type specific environments. At present, there is little evidence for preferences in RFX dimerization patterns [43].

The discovery of new RFX target genes typically starts with searching for X-box motifs, the binding site for RFX TFs. X-box searches have mostly focused on upstream promoter sequences, e.g. upstream of the first exon or of the ATG $[23,27,73]$. Here we expand by relating X-box position to both upstream and downstream of human gene TSS locations. X-box position, motif sequence and conservation across species (cf. Henriksson et al. [74]) allow for a precise ranking of hits. With respect to a given gene TSS location we have assigned these hits to a permissive window $(-2300$ to +1400$)$ and a robust window $(-500$ to + $500)$ for the higher ranks. Our data strengthen and surpass previous work in other organisms where functional X-box motifs were found close to the gene start sites [75]. Our type of analysis will enhance the prediction power of future searches for functional X-box motifs, because relating Xbox motifs to both upstream and downstream of TSS locations of candidate human RFX target genes adds another level of precision to the search procedure. Functional Xbox motifs at larger distances from TSS locations (e.g at distal enhancers) are likely to be the exception rather than the norm. The presence of X-box motifs was shown to contribute to the activeness of both promoters and enhancers, whereby distal enhancers that harbor X-box motifs exhibited greater promoter activity than enhancers that lack them [76]. This phenomenon would fit a model where (as found in Xenopus leavis) Rfx 2 and Foxj1 coordinately regulate ciliary gene expression, with Rfx2 stabilizing Foxj1 binding at chromatin loops [77].

Comparative tissue and cell type specific expression profile clustering represents a complementary approach to Xbox searches for the ascertainment of cross-connections between RFX genes and candidate sets of downstream target genes. We have used this approach successfully to describe the key roles that defined RFX family members play by regulating only certain target genes in e.g. human testis and the gastrointestinal tract. Combining both methods, Xbox searches and expression profile clustering, will be very helpful for the discovery of precise sets of RFX target genes in many different human tissue and cell types.

Studies in mammals suggest that RFX TFs function in terminal cell differentiation or in the maintenance of certain functional specializations. Examples include the differentiation and maintenance of pancreatic $\beta$-cells as insulin producers [78], the repression of collagen formation during adult life [79], the maintenance of testis cord integrity [80], the regulation of spermiogenesis and sperm flagellum assembly [17], the maintenance of postnatal auditory hair cells [81], and the regulation of ciliary genes involved in the assembly and maintenance of functional cilia [16]. Interestingly, RFX TFs seem to exert their function on structures connected to polarized cell surfaces, e.g. cilia, immune synapse, neuronal synapse and the vascular face of $\beta$-cells [82].

Given such a range of RFX TF functions in different tissue and cell types, elucidating their role in disease will be facilitated when more precise connections can be established between specific RFX protein isoforms, RFX target gene sets and quantity or cell type of expression. So far only RFX5 and RFX6 mutations have been linked to defined diseases, while mutations in other $R F X$ genes may cause more complex, pleiotropic disease symptoms. Embryonic lethality in $R f x 1^{-/-}$mice suggests that Rfx1 function cannot be compensated for [83]. RFX mutations may cause ciliopathies, as RFX TFs directly regulate many ciliary genes in different cell and tissue types. The complexity of ciliopathies arises due to primary cilia being present on most human cell types [84]. Very recently, X-box motifs were shown to overlap with type 2 diabetes risk alleles [85], elevating the importance of understanding X-box motif sequence and position, and X-box containing promoter activity in connection to RFX target gene regulation.

Our exhaustive and in-depth characterization of the functional domain composition and the expression profiles of all the eight human $R F X$ genes, including upstream regulatory and downstream target gene analysis, in connection with mammalian studies, e.g. investigating $R f x$ mice mutants, will serve as the basis for uncovering and understanding phenotypes or pathologies of RFX mutations in humans. For example, one might expect male sterility to be associated with mutations in testis specific RFX1-4 gene isoforms, or with dys-regulation of testis specific RFX target genes (e.g. GPR56 [86], ALMS1 [87] and $R F X 1$ ), or with the role upstream $R F X$ regulators (e.g. ESR1 $[88,89])$ play in ciliogenesis.

\section{Conclusions}

We provide a comprehensive and systematic characterization of the expression profiles of all the eight human RFX genes, including the previously undescribed $R F X 8$. We open the window to their potential upstream regulators during development. We advance on how 
human RFX TFs regulate their target genes. Thereby, our study contributes to the understanding of the different functions for RFX TFs in their specific spatial and temporal context in the different tissue and cell types of humans. Our work will greatly help in uncovering their cell-type specific target gene batteries, essential for elucidating RFX-associated aspects of cellular specialization and terminal functional differentiation. In turn, this will aid in understanding disease mechanisms and outcome.

\section{Methods}

\section{Extraction and analysis of CAGE TSS locations from the FANTOM5 database}

CAGE TSS locations and expression profiles were extracted from FANTOM5 Phase I as downloaded from SSTAR [90]: http://fantom.gsc.riken.jp/5/sstar/Main_Page. FANTOM5 TSS data represent expression profiles from 889 biological samples with assigned detection levels in arbitrary units "tags per million" (TPM) [33]. We categorized all samples into three separate groups: human tissues (135 samples $80 \%$ adult and $20 \%$ fetal), human primary cells ( 170 samples - here represented as the average of the donor replicates) and human cell lines (255 samples), and excluded the time course samples. TSS data were extracted and analyzed, and then named with shorthand $\mathrm{p}$ (for promoter) in alphabetical order (pA, pB, pC, etc.) based on the following criteria: (1) if the tissue correlation is equal to or greater than 0.7 and individual TSS locations fall within $100 \mathrm{bp}$ of each other, they were merged into one TSS; (2) if the highest tissue sample TPM is $<1$, this TSS was disregarded unless the highest primary cell (in any donor replicate) or cell line TPM is $\geq 5$; (3) the alphabetical order of TSS locations is based on the descending order of its total sum of TPM values in all 889 biological samples after conditions (1) and (2) are met.

A given TSS location is considered as being expressed broadly if it is expressed at TPM $>5$ in a large number and variety of tissues $(n>10)$. Conversely, a given TSS location is considered as being expressed specifically if it is expressed at TPM $>5$ in a small number of tissues of the same organ $(n<10)$. The exceptions are: (i) pA of RFX4 displays high expression in many tissues $(n>10)$ but specifically in the brain and spinal cord; (ii) RFX8 TSS locations are either lowly expressed $(\mathrm{TPM}<5)$ or at background noise levels $(\mathrm{TPM}<1)$. Additional information about these and other CAGE TSS locations present in the FANTOM5 database (e.g. the presence or absence of TATA boxes, CpG islands, etc.) has been described by Lizio et al. 2015 [90]. All the genomic coordinates are stated in BED format.

\section{Transcript validation from novel TSS locations by RT-PCR} A given TSS location was deemed to be a novel transcript isoform for experimental validation when it does not overlap with or is not found within $+/-50$ bp of the start site (indicated as exon 1) of known protein-coding transcripts with complete open reading frame description in the Ensembl database (release 81 - July 2015, http://www.ensembl.org/). We designed forward primers to bind either within the novel TSS sequence, or overlapping with the $3^{\prime}$ end of the TSS, or at the most $50 \mathrm{bp}$ downstream from the TSS. Reverse primers were designed to always bind downstream of the ATG, respectively, from the reference Ensembl transcript. In the case of the RFX8 gene, we designed additional primers that sandwiched the DBD exonic region to confirm the presence or absence of a DBD-encoding exon. We reverse transcribed $1 \mu \mathrm{g}$ commercial human testis total RNA (Clontech, Cat. No. 636533) and human whole brain total RNA (Clontech, Cat No. 636530) using Invitrogen SuperScript III First-strand Synthesis Super Mix for qRT-PCR (Cat No. 11752-050). We used undiluted cDNA and 40 PCR cycles with the exception of 45 PCR cycles for RFX8. $2 \mu \mathrm{l}$ of the PCR product were cloned using a TOPO TA Cloning Kit (Invitrogen Dual Promoter PCR II-TOPO Vector, Cat No. 450640). Then, 410 white colonies from AMP + IPTG/X-gal plates were screened by PCR M13 vector primers, out of which 2-4 independent samples were sequenced with T7 and SP6 universal primers. Sequencing results were analyzed using the BLAT Tool (UCSC Genome Browser, http:// genome-euro.ucsc.edu/). In the case of the RFX8 DBD transcript validation, at least 100 white colonies were screened with PCR M13 vector primers prior to sequencing, given the overall low expression of the RFX8 gene. Sequences of primers and verified transcripts are listed in Tables S8 and S9, respectively, in Additional file 3.

\section{Determination of RFX protein domains}

Peptide sequences of human RFX1-3 protein domains (AD, DBD, B, C and DIM) as described previously $[9,10]$ were used to determine the corresponding domains in human RFX4-8 using the T-coffee protein sequence alignment program [91] (http://www.tcoffee.org/). Visualization of the RFX transcripts in Fig. 1a with the protein domain composition was done using IBS software [92].

\section{Positional X-box motif scanning}

We scanned for candidate X-box motifs using two known X-box motifs deposited in the JASPAR database [47] (http://jaspar.genereg.net/): human RFX2 (motif MA0600.1, representing a full-site X-box) and RFX5 (motif MA0510.1, representing a half-site X-box). For these scans we used DNA regions of 5000 bp upstream $(-5000)$ and $2000 \mathrm{bp}$ downstream $(+2000)$ as search windows relative to the TSS locations. We selected X-box motifs in the promoter regions, which were captured by the JASPAR built-in scan function (version 5.0_ALPHA) with an 80\% threshold. Previously validated X-box motifs were found with these 
criteria and also independently using the MEME Suite FIMO software (version 4.10.0) [48] (http://meme-suite.org/tools/fimo) with a $p$-value $<0.0001$. Positional motif enrichment was ascertained by analyzing in the same way 10 times random TSS sets from all the CAGE TSS locations present in FANTOM5. The graphical smoothing method employed was local polynomial regression fitting (loess) constructed by the $R$ package ggplot2::geom_smooth [93] with a confidence interval (C.I.) level $=0.95$.

\section{Multiple TF binding profile analysis for the prediction of candidate RFX regulators}

We performed TF binding profile enrichment analyses using the oPOSSUM3 tool [50] with the CORE vertebrate TF binding profiles present in the JASPAR 2016 database [47]. DNA regions of -5000 to +2000 bp of the 30 RFX TSS locations and -200 to $+200 \mathrm{bp}$ from the midpoints of 13 candidate RFX enhancers were used as search windows (foreground). Candidate RFX enhancers were chosen by selecting enhancers present within $-500 \mathrm{~kb}$ to $+500 \mathrm{~kb}$ of the 30 RFX TSS locations, as extracted from Andersson et al. [49] (http://fantom.gsc.riken.jp/5/datafiles/latest/extra/ Enhancers/), and whose expressions were significantly correlated (Spearman correlation with multiple testing correction, False Discovery Rate $<0.05$ ) with RFX TSS locations based on FANTOM5 CAGE expression values (TPM) in 889 biological samples). As background we considered 10-fold larger sets of DNA regions with \%GC matching the ones of the foreground sequences and derived for regions surrounding all phase 1.3 CAGE peak coordinates (http://fantom.gsc .riken.jp/5/datafiles/phase1.3/extra/CAGE_peaks/hg19.cage_ peak_coord_permissive.bed.gz; $-5000 \mathrm{bp}$ and $+2000 \mathrm{bp}$ ) and phase 2.0 enhancer coordinates (http://fantom.gsc.riken .jp/5/datafiles/phase2.0/extra/Enhancers/human_permissive _enhancers_phase_1_and_2.bed.gz; +/- 200 bp) using BiasAway (https://www.ncbi.nlm.nih.gov/pubmed/24927817). We computed the mean $(m)$ and standard deviation $(s d)$ of the distribution of all the $\mathrm{z}$-scores (considering the enrichment of the total number of predicted TFBSs) obtained from oPOSSUM3 and put a threshold at $m+2 \times s d$.

\section{Validation of candidate RFX regulators by siRNA knockdown and qRT-PCR}

SP2 and ESR1 siRNA concentrations (Table S10 in Additional file 3) were optimized for knockdown efficiency (cutoff: more than 2-fold) using qRT-PCR. siRNA and qPCR primer sequences (obtained from Eurofins Genomics: https://www.eurofinsgenomics.eu/) were selected to target all the known protein-coding transcript isoforms. Primer specificities were tested first by common PCR and later by $\mathrm{qPCR}$ analyses of the melting curves using two negative controls, a water sample and a cDNA sample without reverse transcriptase. Sequences of qPCR primers with their amplification efficiencies determined in a standard control setup are listed in Table S11 in Additional file 3. The MCF7 breast cancer cell line (Michigan Cancer Foundation) was used as the human cell line listed in the FANTOM5 database as having sufficiently high expression of both the candidate and the RFX genes (TPM > 5). MCF7 cells were maintained using DMEM $1 \mathrm{~g} / \mathrm{L}$-D-glucose with added pyruvate, $10 \%$ FBS, 1\% Penicillin/Streptomycin and $1 \% \mathrm{~L}$-glutamine at $37^{\circ} \mathrm{C}$ at $5 \% \mathrm{CO}^{2}$. Cells were seeded $24 \mathrm{~h}$ prior to transfection $(150,000$ cells in $2 \mathrm{ml}$ in a 6-well plate format). Lipofectamine RNAiMAX (Invitrogen, Cat. No. 13778-030) was mixed with siRNA according to the manufacturer's instructions. RNA was extracted (RNeasy Mini Kit and DNase Set, QIAGEN) 24 h after transfection and we used 2 biological replicates repeated on three different days of transfection. We converted $1 \mu \mathrm{g}$ RNA to cDNA (Invitrogen SuperScript III First-strand Synthesis Super Mix for qRT-PCR, Cat No. 11752-050). qPCR was performed for 40 cycles in singleplex technical triplicates using FastStart Universal SYBR Green Master with ROX reference dye (Roche Cat No. 04913914001) on an AB7500 Fast machine. We used $2 \mu \mathrm{l}$ of 1:3 diluted cDNA from a biological replicate in $10 \mu \mathrm{l}$ total. Ct levels with automatic threshold were obtained (Additional file 5) and efficiencyadjusted fold-changes were calculated against scrambled (Scr) control siRNA with the geometric mean of HPRT1 and $H S P C B$ as two independent reference genes for normalization [51]. Graph and statistical tests were performed in $R$ using the ggplot2 package [93] and two-tailed one-sample Student's t-test [94].

\section{siRNA knockdown confirmation by immunoblotting}

At $24 \mathrm{~h}$ after transfection with siRNAs, MCF7 cells were collected and washed twice with PBS. Whole cell lysates were prepared upon sonicating the cells in RIPA buffer (Sigma-Aldrich, St. Louis, MO, USA) containing PMSF (1 mM, final concentration) and a protease inhibitor cocktail (Sigma-Aldrich, St. Louis, MO, USA). The protein content of these cell lysates was determined using the BCA protein assay kit (Thermo Scientific, Sweden). A total of $40-80 \mu \mathrm{g}$ of protein was loaded per well, separated on a $12 \%$ SDS-PAGE gel (Bio-Rad, Stockholm, Sweden) and transferred to a $0.45 \mu \mathrm{m}$ pore-sized PVDF membrane (BioRad, Stockholm, Sweden). After transfer, membranes were incubated overnight at $4{ }^{\circ} \mathrm{C}$ with primary antibodies (rabbit polyclonal ESR1, Catalog \# sc-543, dilution - 1:500, Santa Cruz Biotech; rabbit polyclonal SP2 (A-8), Catalog \# sc17,814, Lot \# D0605, dilution - 1:100, Santa Cruz Biotech; rabbit polyclonal beta-tubulin, Catalog \# ab6046, dilution 1:5000, Abcam; mouse monoclonal vinculin, clone V284, Lot \# 2627627, dilution - 1:5000, Millipore) diluted in 5\% milk. Subsequently, blots were washed and incubated with either horseradish peroxidase-conjugated secondary antibody (polyclonal rabbit anti-mouse/HRP, Lot \# 00054403, dilution - 1:3000, Dako Chemicals) or Li-Cor donkey anti- 
mouse IRDye 800CW (Catalog \# 926-32,212, for vinculin) or Li-Cor donkey anti-rabbit IRDye 680LT (Catalog \# 92668,023, for ESR1) for $1 \mathrm{~h}$ at room temperature. Imaging was performed using a Li-Cor Odyssey Fc system. An enhanced chemiluminescence technique (WesternBright Sirius ECL substrate, Advansta) was applied for developing the SP2 blot due to low abundance of the target protein. In all other cases, fluorescence signals were acquired. Band intensities were quantified using ImageJ and normalized with the indicated loading controls.

\section{Human reference sequence}

The human reference sequence used is the Human Feb. 2009 (GRCh37/hg19) Assembly.

\section{Additional files}

Additional file 1: Detailed expression values (TPM) for RFX TSS locations. Expression values in tags per million (TPM) for all 30 RFX TSS locations in all 889 biological samples and their categorization into tissues (135), primary cells (473 donor replicates and 170 merged replicates from the average TPM value of the donor replicates), cell lines (255) and time courses (26). (XLSX $355 \mathrm{~kb}$ )

Additional file 2: Hierarchical clustering, expression plots and top 10 tissues, primary cells and cell lines of RFX TSS locations. Hierarchical clustering of 30 RFX TSS locations (with shorthand $\mathrm{p}$ for promoter) based on expression values (TPM) across 135 human tissue samples, using a 1-Pearson correlation distance measure and average linkage method, as computed by the pvclust $R$ package with nboot $=1000$ with the numbers representing approximately unbiased (au) $p$-values (Suzuki and Shimodaira, 2006). Tissue clusters are color-coded and represent the groups of tissues with the highest overall expression values: immune system (teal), gastrointestinal tract (purple), testis (green), brain and spinal cord (red), and two minor clusters, uterus and lung (black). RFX TSS locations without color code have low expression values (TPM $<5)$. This is followed by the expression profiles of 30 RFX TSS locations in human tissues, primary cells and cell lines, whereby for every one of the eight human RFX genes (1-8), summarized TSS profile data are presented vertically ("top-down"), starting with the a tissue plot, followed by a table of the top 10 tissues, a table of the top 10 primary cells and a table of the top 10 cell lines (highest expression levels are listed first, respectively). The tissue plot is the expression level in log (base 10) TPM against tissues that are sorted from the highest to the lowest expressed from 135 tissues, whereby the plot only includes the first 100 tissues. The arbitrary unit for detection of expression is tags per million (TPM) as defined by FANTOM5. We consider TPM $<5$ to be lowly expressed and TPM $<1$ to be background noise. (PDF $3276 \mathrm{~kb}$ )

Additional file 3: Supporting tables, figures and supplementary references. Table $\mathbf{S 1}$. Summary of RFX1-8 expression data and novel transcript validation. Table S2. Positions of functional domains encoded by RFX transcripts. Table S3. Experimentally proven, direct RFX target genes in humans from the literature. Table S4. Human X-box motifs selected from the JASPAR database. Table S5. Experimentally validated human X-box motif sequences in promoter regions that were captured by the scanning criteria. Table S6. Experimentally validated human X-box motif sequences that were either in distal regions or that were not captured by the scanning criteria. Table S7. RFX correlated enhancers within $+/-500 \mathrm{~kb}$ of RFX TSS locations. Table S8. Primer sequences for novel RFX transcripts validation. Table S9. Verified novel RFX transcript sequences. Table S10. siRNA sequences for candidate RFX regulators. Table S11. qPCR primer sequences and amplification efficiencies for validation of candidate RFX regulators. Figure S1. Human RFX1-8 DBD protein sequence alignment. Supplementary references. (DOCX 424 kb)
Additional file 4: Detailed candidate RFX regulator oPOSSUM3 scanning results using JASPAR 2016 core vertebrate TF binding profiles. Transcription factor binding sites (TFBS) scanning results from oPOSSUM3 within the promoter and enhancer regions of RFX1-8 using the CORE vertebrate TF binding profiles in JASPAR 2016. Included are the DNA regions that were considered as foreground and the following TF binding site details: SP2 (specificity protein 2) (JASPAR profile MA0516.1) and ESR1 (estrogen receptor alpha) (MA0112.3). (XLSX 50 kb)

Additional file 5: $\mathrm{Ct}$ levels of qRT-PCR, used for validation of candidate $R F X$ regulators by siRNA knockdown. Individual $C t$ levels with automatic threshold obtained on an AB7500 Fast machine for SP2 and ESR1 as candidate RFX regulators and their respective test siRNA and scrambled (SCr) control siRNA knockdown data on RFX genes (RFX1, RFX2, RFX3, RFX5, RFX7) and the two reference genes (HPRT1, HSPCB). (XLSX $33 \mathrm{~kb})$

\section{Abbreviations}

AD: Activating domain; B: B domain; C: C domain; CAGE: Cap Analysis of Gene Expression; DBD: DNA binding domain; DIM: Dimerization domain; FANTOM5: Functional Annotation of Mammalian Genome 5; RFX: Regulatory Factor binding to the X-box; TF: Transcription factor; TFBS: Transcription factor binding site; TPM: Tags per million; TSS: Transcription start site

\section{Acknowledgements}

We express gratitude toward the FANTOM Consortium and its public database (http://fantom.gsc.riken.jp/). We thank Min Jia from the Karolinska Institute (KI) Department of Biosciences and Nutrition for providing the MCF7 cell line and Arun Selvam from the KI Department of Laboratory Medicine for assistance in protein work.

\section{Funding}

We acknowledge financial support from the Swedish Research Council (Vetenskapsrådet), from the Swedish Brain Foundation (Hjärnfonden), and from the Torsten Söderberg and Åhlén Foundations. DST received support from the $\mathrm{KI}$ in the form of a PhD student (KID) scholarship. MV was supported by the EU Horizon 2020 Marie Curie Individual Fellowship. AM was supported by a Genome Canada Large Scale Applied Research Grant (No. 174CDE), by funding provided by the Child and Family Research Institute and the British Columbia Children's Hospital Foundation (Vancouver, BC, Canada), by funding from the Norwegian Research Council (Helse Sør-Øst) and the University of Oslo through the Centre for Molecular Medicine Norway (NCMM) and the Oslo University Hospital (Radiumhospitalet). GL acknowledges fellowship support from the Swedish Society for Medical Research (Svenska Sällskapet för Medicinsk Forskning), the Lars Hierta Memorial Foundation (Stiftelsen Lars Hiertas Minne) and from the Thuring Foundation. JK was the recipient of a KI Distinguished Professor Award and a Royal Society Wolfson Research Excellence Award. PS received support from the KI Strategic Neurosciences Program.

\section{Availability of data and materials}

All data generated or analysed during this study are included in this published article (and its additional files).

\section{Authors' contributions}

PS, JK and COD conceived and supervised the study. DST performed the database work, data analysis, experimental validation work and made all the Figures. MV contributed to the database work and data analysis presented in Table 1, Fig. 3 and Fig. 4. EMJ contributed to the experimental validation work presented in Table 1 and Fig. 5. AM performed the database work and data analyses presented in Fig. 4. GL contributed to the database work and data analysis presented in Fig. 2. SM and GL performed and analyzed all the protein work. DST, JK and PS wrote and edited the manuscript. All authors read and approved the final version of the manuscript.

\section{Ethics approval and consent to participate}

For work with mammalian cell cultures the authors are in possession of the applicable permits for carrying out studies with genetically modified micro-organisms / GMMs (Dnr 5.8.18-1012/14; Dnr 5.5.18-6998/15).

\section{Consent for publication}

This section is not applicable. 


\section{Competing interests}

The authors declare that they have no competing interests.

\section{Publisher's Note}

Springer Nature remains neutral with regard to jurisdictional claims in published maps and institutional affiliations.

\section{Author details}

'Department of Biosciences and Nutrition, Karolinska Institutet, Huddinge, Sweden. ${ }^{2}$ Department of Biology, Bioinformatics Centre, Section for Computational and RNA Biology, University of Copenhagen, Copenhagen, Denmark. ${ }^{3}$ Department of Medical Genetics, Centre for Molecular Medicine and Therapeutics at the Child and Family Research Institute, University of British Columbia, Vancouver, Canada. ${ }^{4}$ Centre for Molecular Medicine Norway (NCMM), Nordic EMBL partnership, University of Oslo, Oslo, Norway. ${ }^{5}$ Department of Cancer Genetics, Institute for Cancer Research, Oslo University Hospital Radiumhospitalet, Oslo, Norway. ${ }^{6}$ Department of Laboratory Medicine, Karolinska Institutet, Huddinge, Sweden. ${ }^{7}$ Science for Life Laboratory, Karolinska Institutet, Stockholm, Sweden. ${ }^{8}$ School of Basic and Medical Biosciences, King's College London, London, UK. ${ }^{9}$ Folkhälsan Institute of Genetics and Molecular Neurology Research Program, University of Helsinki, Helsinki, Finland.

\section{Received: 3 November 2017 Accepted: 21 February 2018}

\section{Published online: 06 March 2018}

\section{References}

1. Gajiwala KS, Chen H, Cornille F, Roques BP, Reith W, Mach B, Burley SK Structure of the winged-helix protein hRFX1 reveals a new mode of DNA binding. Nature. 2000;403:916-21.

2. Piasecki BP, Burghoorn J, Swoboda P. Regulatory factor X (RFX)-mediated transcriptional rewiring of ciliary genes in animals. Proc Natl Acad Sci. 2010; 107(29):12969-74.

3. Durand B, Vandaele C, Spencer D, Pantalacci S, Couble P. Cloning and characterization of dRFX, the drosophila member of the RFX family of transcription factors. Gene. 2000:246(1-2):285-93.

4. Otsuki K, Hayashi Y, Kato M, Yoshida H, Yamaguchi M. Characterization of dRFX2, a novel RFX family protein in drosophila. Nucleic Acids Res. 2004; 32(18):5636-48.

5. Reith W, Herrero-Sanchez C, Kobr M, Silacci P, Berte C, Barras E, Fey S, Mach B. MHC class II regulatory factor RFX has a novel DNA binding domain and functionally independant dimerization domain. Genes Dev. 1990;4:1528-40.

6. Reith W, Ucla C, Barras E, Gaud A, Durand B, Herrero-Sanchez C, Kobr M, Mach B. RFX1, a transactivator of hepatitis B virus enhancer I, belongs to a novel family of homodimeric and heterodimeric DNA-binding proteins. Mol Cell Biol. 1994;14(2):1230-44.

7. Emery $\mathrm{P}$, Durand $\mathrm{B}$, Mach $\mathrm{B}$, Reith $\mathrm{W}$. RFX proteins, a novel family of DNA binding proteins conserved in the eukaryotic kingdom. Nucleic Acids Res. 1996:24:803-7.

8. Swoboda P, Adler HT, Thomas JH. The RFX-type transcription factor DAF-19 regulates sensory neuron cilium formation in C. elegans. $\mathrm{Mol}$ Cell. 2000:5(3):411-21.

9. Aftab S, Semenec L, Chu J, Chen N. Identification and characterization of novel human tissue-specific RFX transcription factors. BMC Evol Biol. 2008; $8(1): 226$

10. Chu J, Baillie D, Chen N. Convergent evolution of RFX transcription factors and ciliary genes predated the origin of metazoans. BMC Evol Biol. 2010; 10(1):130.

11. Zaim J, Speina E, Kierzek AM. Identification of new genes regulated by the Crt1 transcription factor, an effector of the DNA damage checkpoint pathway in Saccharomyces cerevisiae. J Biol Chem. 2005;280(1):28-37.

12. Garg A, Futcher B, Leatherwood J. A new transcription factor for mitosis: in Schizosaccharomyces pombe, the RFX transcription factor Sak1 works with forkhead factors to regulate mitotic expression. Nucleic Acids Res. 2015; 43(14):6874-88

13. Reith $W$, Mach $B$. The bare lymphocyte syndrome and the regulation of MHC expression. Annu Rev Immunol. 2001:19:331-73.

14. Senti G, Swoboda P. Distinct isoforms of the RFX transcription factor DAF-19 regulate Ciliogenesis and maintenance of synaptic activity. Mol Biol Cell. 2008;19(12):5517-28
15. Senti G, Ezcurra M, Löbner J, Schafer WR, Swoboda P. Worms with a single functional sensory cilium generate proper neuron-specific behavioral output. Genetics. 2009;183(2):595-605.

16. Choksi SP, Lauter G, Swoboda P, Roy S. Switching on cilia: transcriptional networks regulating ciliogenesis. Development. 2014;141(7):1427-41.

17. Wu Y, Hu X, Li Z, Wang M, Li S, Wang X, Lin X, Liao S, Zhang Z, Feng X, et al. Transcription factor RFX2 is a key regulator of mouse Spermiogenesis. Sci Rep. 2016;6:20435.

18. Magnani D, Morle L, Hasenpusch-Theil K, Paschaki M, Jacoby M, Schurmans S, Durand $\mathrm{B}$, Theil T. The ciliogenic transcription factor Rfx3 is required for the formation of the thalamocortical tract by regulating the patterning of prethalamus and ventral telencephalon. Hum Mol Genet. 2015;24(9):2578-93.

19. Baas D, Meiniel A, Benadiba C, Bonnafe E, Meiniel O, Reith W, Durand B. A deficiency in RFX3 causes hydrocephalus associated with abnormal differentiation of ependymal cells. Eur J Neurosci. 2006;24:1020-30.

20. Ait-Lounis A, Baas D, Barras E, Benadiba C, Charollais A, Nlend Nlend R, Liegeois D, Meda P, Durand B, Reith W. Novel function of the ciliogenic transcription factor RFX3 in development of the endocrine pancreas. Diabetes. 2007:56:950-9.

21. El Zein L, Ait-Lounis A, Morlé L, Thomas J, Chhin B, Spassky N, Reith W, Durand B. RFX3 governs growth and beating efficiency of motile cilia in mouse and controls the expression of genes involved in human ciliopathies. J Cell Sci. 2009;122(17):3180-9.

22. Chen N, Mah A, Blacque OE, Chu J, Phgora K, Bakhoum MW, Hunt Newbury CR, Khattra J, Chan S, Go A, et al. Identification of ciliary and ciliopathy genes in Caenorhabditis elegansthrough comparative genomics. Genome Biol. 2006;7(12):R126.

23. Laurencon A, Dubruille R, Efimenko E, Grenier G, Bissett R, Cortier E, Rolland $\checkmark$, Swoboda P, Durand B. Identification of novel regulatory factor X (RFX) target genes by comparative genomics in drosophila species. Genome Biol. 2007:8(9):R195.

24. Thomas J, Morlé L, Soulavie F, Laurençon A, Sagnol S, Durand B. Transcriptional control of genes involved in ciliogenesis: a first step in making cilia. Biol Cell. 2010;102(9):499-513.

25. Emery P, Strubin M, Hofmann K, Bucher P, Mach B, Reith W. A consensus motif in the RFX DNA binding domain and binding domain mutants with altered specificity. Mol Cell Biol. 1996;16(8):4486-94.

26. Blacque OE, Perens EA, Boroevich KA, Inglis PN, Li C, Warner A, Khattra J, Holt RA, Ou G, Mah AK, et al. Functional genomics of the cilium, a sensory organelle. Curr Biol. 2005;15:935-41.

27. Efimenko E, Bubb K, Mak HY, Holzman T, Leroux MR, Ruvkun G, Thomas JH, Swoboda P. Analysis of xbx genes in C. Elegans. Development. 2005;132(8):1923-34

28. Jolma A, Yan J, Whitington T, Toivonen J, Nitta Kazuhiro R, Rastas P, Morgunova E, Enge M, Taipale M, Wei G, et al. DNA-binding specificities of human transcription factors. Cell. 2013;152(1-2):327-39.

29. Beckers A, Alten L, Viebahn C, Andre P, Gossler A. The mouse homeobox gene Noto regulates node morphogenesis, notochordal ciliogenesis, and left-right patterning. Proc Natl Acad Sci. 2007;104(40):15765-70.

30. Soyer J, Flasse L, Raffelsberger W, Beucher A, Orvain C, Peers B, Ravassard P, Vermot J, Voz ML, Mellitzer G, et al. Rfx6 is an Ngn3-dependent winged helix transcription factor required for pancreatic islet cell development. Development. 2010;137(2):203-12.

31. Cachero S, Simpson TI, zur Lage PI, Ma L, Newton FG, Holohan EE, Armstrong JD, Jarman AP. The gene regulatory Cascade linking proneural specification with differentiation in Drosophila sensory neurons. PLoS Biol. 2011;9(1):e1000568.

32. Huang M, Zhou Z, Elledge SJ. The DNA replication and damage checkpoint pathways induce transcription by inhibition of the Crt1 repressor. Cell. 1998; 94(5):595-605.

33. FANTOM Consortium, RIKEN PMI, CLST (DGT): A promoter-level mammalian expression atlas. Nature 2014, 507(7493):462-470.

34. Rousseau P, Masternak K, Krawczyk M, Reith W, Dausset J, Carosella ED, Moreau $P$ : In vivo, RFX5 binds differently to the human leucocyte antigen- $E$, $-F$, and $-G$ gene promoters and participates in HLA class I protein expression in a cell type-dependent manner. Immunology 2004, 111(1):53-65.

35. Smith SB, Qu H-Q, Taleb N, Kishimoto NY, Scheel DW, Lu Y, Patch A-M, Grabs R, Wang J, Lynn FC, et al. Rfx6 directs islet formation and insulin production in mice and humans. Nature. 2010:463(7282):775-80.

36. Piccand J, Strasser P, Hodson David J, Meunier A, Ye T, Keime C, Birling M-C, Rutter Guy A, Gradwohl G. Rfx6 maintains the functional identity of adult pancreatic $\beta$ cells. Cell Rep. 2014;9(6):2219-32. 
37. Kistler WS, Horvath GC, Dasgupta A, Kistler MK. Differential expression of Rfx1-4 during mouse spermatogenesis. Gene Expr Patterns. 2009;9(7):515-9.

38. Feng C, Li J, Zuo Z. Expression of the transcription factor regulatory factor X1 in mouse brain. Folia Histochemica et Cytobiologica / Polish Academy of Sciences, Polish Histochemical and Cytochemical Society. 2011;49(2):344-51.

39. Manojlovic Z, Earwood R, Kato A, Stefanovic B, Kato Y: RFX7 is required for the formation of cilia in the neural tube. Mech Dev 2014, 132(0):28-37.

40. Chung M-I, Peyrot SM, LeBoeuf S, Park TJ, McGary KL, Marcotte EM, Wallingford JB. RFX2 is broadly required for ciliogenesis during vertebrate development. Dev Biol. 2012;363(1):155-65.

41. La Manno G, Gyllborg D, Codeluppi S, Nishimura K, Salto C, Zeisel A, Borm Lars E, Stott Simon RW, Toledo Enrique M, Villaescusa JC, et al. Molecular Diversity of Midbrain Development in Mouse, Human, and Stem Cells. Cell. 2016;167(2):566-80. e519

42. Didon L, Zwick R, Chao IW, Walters M, Wang R, Hackett N, Crystal R. RFX3 modulation of FOXJ1 regulation of cilia genes in the human airway epithelium. Respir Res. 2013;14(1):70.

43. Morotomi-Yano K, Yano K-I, Saito H, Sun Z, Iwama A, Miki Y. Human regulatory factor $X 4$ (RFX4) is a testis-specific dimeric DNA-binding protein that cooperates with other human RFX members. J Biol Chem. 2002;277(1):836-42.

44. Matsushita H, Uenaka A, Ono T, Hasegawa K, Sato S, Koizumi F, Nakagawa K, Toda M, Shingo T, Ichikawa T, et al. Identification of glioma-specific RFX4-E and $-\mathrm{F}$ isoforms and humoral immune response in patients. Cancer Sci. 2005;96(11):801-9.

45. Zhang D, Zeldin DC, Blackshear PJ. Regulatory factor X4 variant 3: a transcription factor involved in brain development and disease. J Neurosci Res. 2007;85:3515-22.

46. Zhang D, Stumpo DJ, Graves JP, DeGraff LM, Grissom SF, Collins JB, Li L, Zeldin DC, Blackshear PJ. Identification of potential target genes for RFX4 V3, a transcription factor critical for brain development. J Neurochem. 2006; 98(3):860-75.

47. Mathelier A, Fornes O, Arenillas DJ, Chen C-Y, Denay G, Lee J, Shi W, Shyr C, Tan G, Worsley-Hunt R, et al. JASPAR 2016: a major expansion and update of the open-access database of transcription factor binding profiles. Nucleic Acids Res. 2016;44(D1):D110-5.

48. Grant $C E$, Bailey TL, Noble WS. FIMO: scanning for occurrences of a given motif. Bioinformatics. 2011;27(7):1017-8.

49. Andersson R, Gebhard C, Miguel-Escalada I, Hoof I, Bornholdt J, Boyd M, Chen Y, Zhao X, Schmidl C, Suzuki T, et al. An atlas of active enhancers across human cell types and tissues. Nature. 2014;507(7493):455-61.

50. Kwon AT, Arenillas DJ, Hunt RW, Wasserman WW: oPOSSUM-3: Advanced Analysis of Regulatory Motif Over-Representation Across Genes or ChIP-Seq Datasets. G3: Genes|Genomes|Genetics 2012, 2(9):987-1002.

51. Hellemans J, Mortier G, De Paepe A, Speleman F, Vandesompele J. qBase relative quantification framework and software for management and automated analysis of real-time quantitative PCR data. Genome Biol. 2007;8(2):R19.

52. Båvner A, Sanyal S, Gustafsson J-Å, Treuter E. Transcriptional corepression by SHP: molecular mechanisms and physiological consequences. Trends Endocrinol \& Metabolism. 2005;16(10):478-88.

53. Liang H, Xiao G, Yin H, Hippenmeyer S, Horowitz JM, Ghashghaei HT. Neural development is dependent on the function of specificity protein 2 in cell cycle progression. Development. 2013;140(3):552-61.

54. Bondesson M, Hao R, Lin C-Y, Williams C, Gustafsson J-Å. Estrogen receptor signaling during vertebrate development. Biochimica et Biophysica Acta (BBA) - Gene Regulatory Mechanisms. 2015;1849(2):142-51.

55. Lonze BE, Ginty DD. Function and regulation of CREB family transcription factors in the nervous system. Neuron. 2002;35(4):605-23.

56. Whittington N, Cunningham D, Le T-K, De Maria D, Silva EM. Sox21 regulates the progression of neuronal differentiation in a dose-dependent manner. Dev Biol. 2015;397(2):237-47.

57. Wang J, Galvao J, Beach KM, Luo W, Urrutia RA, Goldberg JL, Otteson DC. Novel roles and mechanism for Krüppel-like factor 16 (KLF16) regulation of neurite outgrowth and Ephrin receptor A5 (EphA5) expression in retinal ganglion cells. J Biol Chem. 2016;291(35):18084-95.

58. Quach DH, Oliveira-Fernandes M, Gruner KA, Tourtellotte WG. A sympathetic neuron autonomous role for Egr3-mediated gene regulation in dendrite morphogenesis and target tissue innervation. J Neurosci. 2013;33(10):4570-83.

59. Poirier R, Cheval H, Mailhes C, Garel S, Charnay P, Davis S, Laroche S. Distinct functions of Egr gene family members in cognitive processes. Front Neurosci. 2008;2(1):47-55.
60. Kasberg AD, Brunskill EW, Steven Potter S. SP8 regulates signaling centers during craniofacial development. Dev Biol. 2013;381(2):312-23.

61. Li S, Miao T, Sebastian M, Bhullar P, Ghaffari E, Liu M, Symonds Alistair LJ, Wang $P$. The transcription factors Egr2 and Egr3 are essential for the control of inflammation and antigen-induced proliferation of $B$ and $T$ cells. Immunity. 2012;37(4):685-96.

62. Shahrin NH, Diakiw S, Dent LA, Brown AL, D'Andrea RJ. Conditional knockout mice demonstrate function of Klf5 as a myeloid transcription factor. Blood. 2016;128(1):55-9.

63. Meinders M, Kulu DI, van de Werken HJG, Hoogenboezem M, Janssen H, Brouwer RWW, van ljcken WFJ, Rijkers E-J, Demmers JAA, Krüger I, et al. Sp1/ Sp3 transcription factors regulate hallmarks of megakaryocyte maturation and platelet formation and function. Blood. 2015;125(12):1957-67.

64. Yoshida K, Maekawa T, Zhu Y, Renard-Guillet C, Chatton B, Inoue K, Uchiyama T, Ishibashi K-I, Yamada T, Ohno N, et al. The transcription factor ATF7 mediates lipopolysaccharide-induced epigenetic changes in macrophages involved in innate immunological memory. Nat Immunol. 2015;16(10):1034-43.

65. Ma L, Quigley I, Omran H, Kintner C. Multicilin drives centriole biogenesis via E2f proteins. Genes Dev. 2014;28(13):1461-71.

66. Chen $\mathrm{H}-\mathrm{Z}$, Tsai S-Y, Leone $\mathrm{G}$. Emerging roles of E2Fs in cancer: an exit from cell cycle control. Nat Rev Cancer. 2009;9(11):785-97.

67. Jen J, Wang Y-C. Zinc finger proteins in cancer progression. J Biomed Sci. 2016;23(1):53.

68. Eguchi T, Prince T, Wegiel B, Calderwood SK. Role and regulation of myeloid zinc finger protein 1 in cancer. J Cell Biochem. 2015;116(10):2146-54.

69. Schemmer J, Araúzo-Bravo MJ, Haas N, Schäfer S, Weber SN, Becker A, Eckert D, Zimmer A, Nettersheim D, Schorle H. Transcription factor TFAP2C regulates major programs required for murine fetal germ cell maintenance and Haploinsufficiency predisposes to Teratomas in male mice. PLoS One. 2013;8(8):e71113.

70. Heinrich R, Livne E, Ben-Izhak O, Aronheim A. The c-Jun dimerization protein 2 inhibits cell transformation and acts as a tumor suppressor gene. J Biol Chem. 2004;279(7):5708-15.

71. Shi Y, Zhang L, Song S, Teves ME, Li H, Wang Z, Hess RA, Jiang G, Zhang $Z$. The mouse transcription factor-like 5 gene encodes a protein localized in the manchette and centriole of the elongating spermatid. Andrology. 2013;1(3):431-9.

72. Lubelsky Y, Reuven N, Shaul Y. Autorepression of Rfx1 gene expression: functional conservation from yeast to humans in response to DNA replication arrest. Mol Cell Biol. 2005;25(23):10665-73.

73. Tammimies K, Bieder A, Lauter G, Sugiaman-Trapman D, Torchet R, Hokkanen M-E, Burghoorn J, Castrén E, Kere J, Tapia-Páez I, et al. Ciliary dyslexia candidate genes DYX1C1 and DCDC2 are regulated by regulatory factor (RF) $X$ transcription factors through $X$-box promoter motifs. FASEB J. 2016;30(10):3578-87.

74. Henriksson J, Piasecki BP, Lend K, Bürglin TR, Swoboda P: Chapter Sixteen Finding Ciliary Genes: A Computational Approach. In: Methods in Enzymology. Edited by Wallace FM, vol. 525. Amsterdam: Academic Press; 2013: $327-350$.

75. Burghoorn J, Piasecki BP, Crona F, Phirke P, Jeppsson KE, Swoboda P. The in vivo dissection of direct RFX-target gene promoters in C. Elegans reveals a novel cis-regulatory element, the C-box. Dev Biol. 2012;368(2):415-26.

76. Nguyen TA, Jones RD, Snavely AR, Pfenning AR, Kirchner R, Hemberg M, Gray JM. High-throughput functional comparison of promoter and enhancer activities. Genome Res. 2016;26(8):1023-33.

77. Quigley IK, Kintner C. Rfx2 stabilizes Foxj1 binding at chromatin loops to enable multiciliated cell gene expression. PLoS Genet. 2017;13(1): e1006538.

78. Chandra V, Albagli-Curiel O, Hastoy B, Piccand J, Randriamampita C, Vaillant E, Cavé $H$, Busiah K, Froguel P, Vaxillaire $M$, et al. RFX6 regulates insulin secretion by modulating Ca2+ homeostasis in human $\beta$ cells. Cell Rep. 2014;9(6):2206-18.

79. Sengupta PK, Fargo J, Smith BD. The RFX family interacts at the collagen (COL1A2) start site and represses transcription. J Biol Chem. 2002;277(28):24926-37.

80. Wang B, Qi T, Chen S-Q, Ye L, Huang Z-S, Li H. RFX1 maintains testis cord integrity by regulating the expression of Itga6 in male mouse embryos. Mol Reprod Dev. 2016;83(7):606-14.

81. Elkon R, Milon B, Morrison L, Shah M, Vijayakumar S, Racherla M, Leitch CC, Silipino L, Hadi S, Weiss-Gayet M, et al. RFX transcription factors are essential for hearing in mice. Nat Commun. 2015;6:8549. 
82. Low JT, Zavortink M, Mitchell JM, Gan WJ, Do OH, Schwiening CJ, Gaisano HY, Thorn P. Insulin secretion from beta cells in intact mouse islets is targeted towards the vasculature. Diabetologia. 2014;57(8):1655-63.

83. Feng $C, X u$ W, Zuo Z. Knockout of the regulatory factor $X 1$ gene leads to early embryonic lethality. Biochem Biophys Res Commun. 2009;386(4):715-7.

84. Reiter JF, Leroux MR. Genes and molecular pathways underpinning ciliopathies. Nat Rev Mol Cell Biol. 2017;18(9):533-47.

85. Varshney A, Scott LJ, Welch RP, Erdos MR, Chines PS, Narisu N, Albanus RDO, Orchard P, Wolford BN, Kursawe R, et al. Genetic regulatory signatures underlying islet gene expression and type 2 diabetes. Proc Natl Acad Sci. 2017;114(9):2301-6.

86. Bae B-I, Tietjen I, Atabay KD, Evrony GD, Johnson MB, Asare E, Wang PP, Murayama AY, Im K, Lisgo SN, et al. Evolutionarily dynamic alternative splicing of GPR56 regulates regional cerebral cortical patterning. Science. 2014:343(6172):764-8.

87. Purvis TL, Hearn T, Spalluto C, Knorz VJ, Hanley KP, Sanchez-Elsner T, Hanley NA, Wilson DI. Transcriptional regulation of the Alström syndrome gene ALMS1 by members of the RFX family and Sp1. Gene. 2010;460(1-2):20-9.

88. Nanjappa MK, Hess RA, Medrano TI, Locker SH, Levin ER, Cooke PS. Membrane-localized estrogen receptor 1 is required for normal male reproductive development and function in mice. Endocrinology. 2016; 157(7):2909-19.

89. Hess RA. Small tubules, surprising discoveries: from efferent ductules in the turkey to the discovery that estrogen receptor alpha is essential for fertility in the male. Anim Reprod. 2015;12(1):7-23.

90. Lizio M, Harshbarger J, Shimoji H, Severin J, Kasukawa T, Sahin S, Abugessaisa I, Fukuda S, Hori F, Ishikawa-Kato S, et al. Gateways to the FANTOM5 promoter level mammalian expression atlas. Genome Biol. 2015;16(1):22.

91. Notredame C, Higgins DG, Heringa J. T-coffee: a novel method for fast and accurate multiple sequence alignment. J Mol Biol. 2000;302(1):205-17.

92. Liu W, Xie Y, Ma J, Luo X, Nie P, Zuo Z, Lahrmann U, Zhao Q, Zheng Y, Zhao $Y$, et al. IBS: an illustrator for the presentation and visualization of biological sequences. Bioinformatics. 2015;31(20):3359-61.

93. Wickham H: ggplot2: Elegant Graphics for Data Analysis, vol. VIII, 213: SpringerVerlag New York; 2009.

94. R Development Core Team: R: A Language and Environment for Statistical Computing. www.R-project.org/.. R Foundation for Statistical Computing; 2016. Accessed 20 Sept 2017.

95. Warnes GR, Bolker B, Bonebakker L, Gentleman R, Huber W, Liaw A, Lumley T, Maechler M, Magnusson A, Moeller S et al: gplots: Various R Programming Tools for Plotting Data. R package version 3.0.1. https://CRAN.R-project.org/ package=gplots; 2016. Accessed 28 Mar 2017.

\section{Submit your next manuscript to BioMed Central and we will help you at every step:}

- We accept pre-submission inquiries

- Our selector tool helps you to find the most relevant journal

- We provide round the clock customer support

- Convenient online submission

- Thorough peer review

- Inclusion in PubMed and all major indexing services

- Maximum visibility for your research

Submit your manuscript at www.biomedcentral.com/submit 